\title{
Detection of post-harvest fungal diseases of mango by X-ray scanning non-destructive technology
}

\section{Gadgile Dhondiram $^{1 *}$ and Chavan Ashok ${ }^{2}$}

\author{
${ }^{1}$ Department of Botany, Madhavrao Patil College, Palam Tq- Palam Dist. Parbhani (M.S.), India \\ ${ }^{2}$ Department of Botany, Dr. Babasaheb Ambedkar Marathwada University, Aurangabad-431004. (M.S.), India.
}

Gadgile D, Chavan A 2017 - Detection of post-harvest fungal diseases of mango by X-ray scanning non-destructive technology. Plant Pathology \& Quarantine 7(1), 65-69, Doi $10.5943 / \mathrm{ppq} / 7 / 1 / 8$.

\begin{abstract}
The detection of Aspergillus niger rot, anthracnose and Rhizopus rot infection in post-harvest mango fruit was demonstrated by a non-destructive X-ray scanning technique carried out 4 and 5 days post inoculation. It is suggested that such technology for detection of fungal infection may be useful as an imaging-based mango sorting system.
\end{abstract}

Key words - anthracnose - Aspergillus niger rot - Mangifera indica - novel technique - Rhizopus rot

\section{Introduction}

X-ray technology has been applied regularly in industry, hospitals, airport security, etc. Early infestation caused by fruit fly in apple, pear, peach, cherry tomato and orange can be detected by Xray scanning (Yang et al. 2006). Spongy tissue of mango fruits can be detected by X-ray scanning (Thomas et al. 1993, Janave 2007). However, there are no references of X-ray scanning technology being used to detect fungal infection in post-harvest fruits of mango. Hence, the aim of this research was to demonstrate the detection of fungal infection of mango fruit by X-ray scanning non-destructive technology.

\section{Materials \& Methods}

Spore suspensions of Aspergillus niger, Colletotrichum gloeosporioides and Rhizopus stolonifer were prepared and inoculated into same sized mango fruits (Kesar variety) using sterilized disposable syringes in aseptic conditions. Healthy mango without inoculation was used as control. Four and 5 days following inoculation, the fruits were scanned by a digital X-ray system. The specifications with which $\mathrm{X}$-ray exposure was taken were $\mathrm{kv}=63, \mathrm{~mA}=160$ and $\mathrm{mAs}=13$.

\section{Results}

Four and 5 days after inoculation, Aspergillus niger, Colletotrichum gloeosporioides and Rhizopus stolonifer fungal infection of mango fruit could be detected by X-ray scanning. Infested fruit showed dark areas in the fungal infected portion, while uninfected fruit showed a uniform light 
grey area (Figs 1-3). After X-ray scanning, when infected fruits were cut, infection was seen clearly.

\section{Discussion}

To our knowledge, this is the first reference for detection of post-harvest fungal disease in fruit. X-ray scanning non-destructive technology for detection of fungal infection may be useful as an imaging-based mango sorting system. It may be useful for traders exporting bulk quantities of fruits.
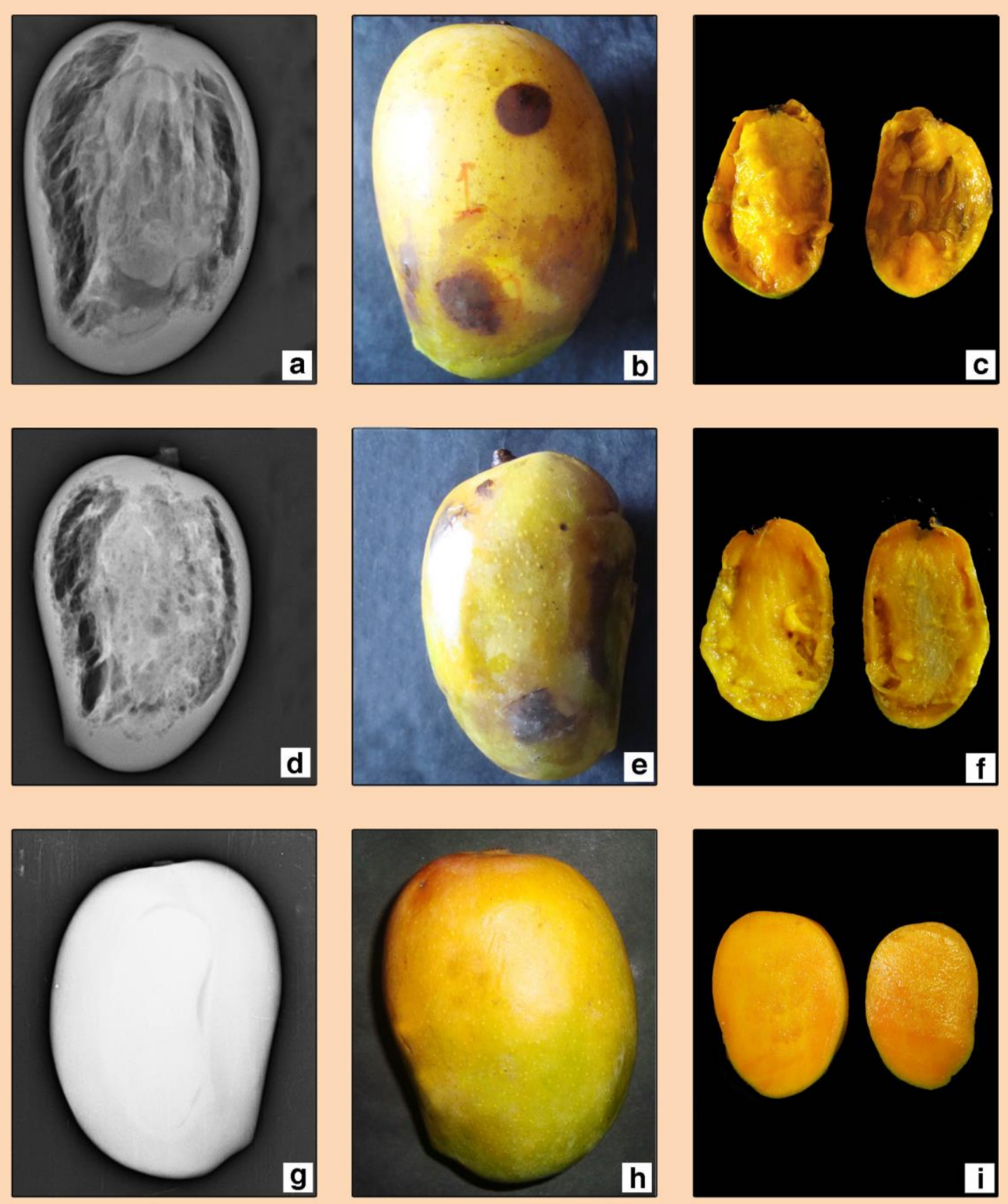

Fig. 1 - X-ray scanning of black mould rot (Aspergillus niger rot) of mango fruit. a. X-ray image 4 days post-inoculation, b. uncut fruit 4 days post-inoculation, c. cut fruit 4 days post-inoculation, $d$. $\mathrm{X}$-ray image 5 days post-inoculation, e. uncut fruit 5 days post-inoculation, f. cut fruit 5 days postinoculation, g. X-ray image of healthy fruit, h. Healthy uncut mango, i. Healthy cut fruit. 

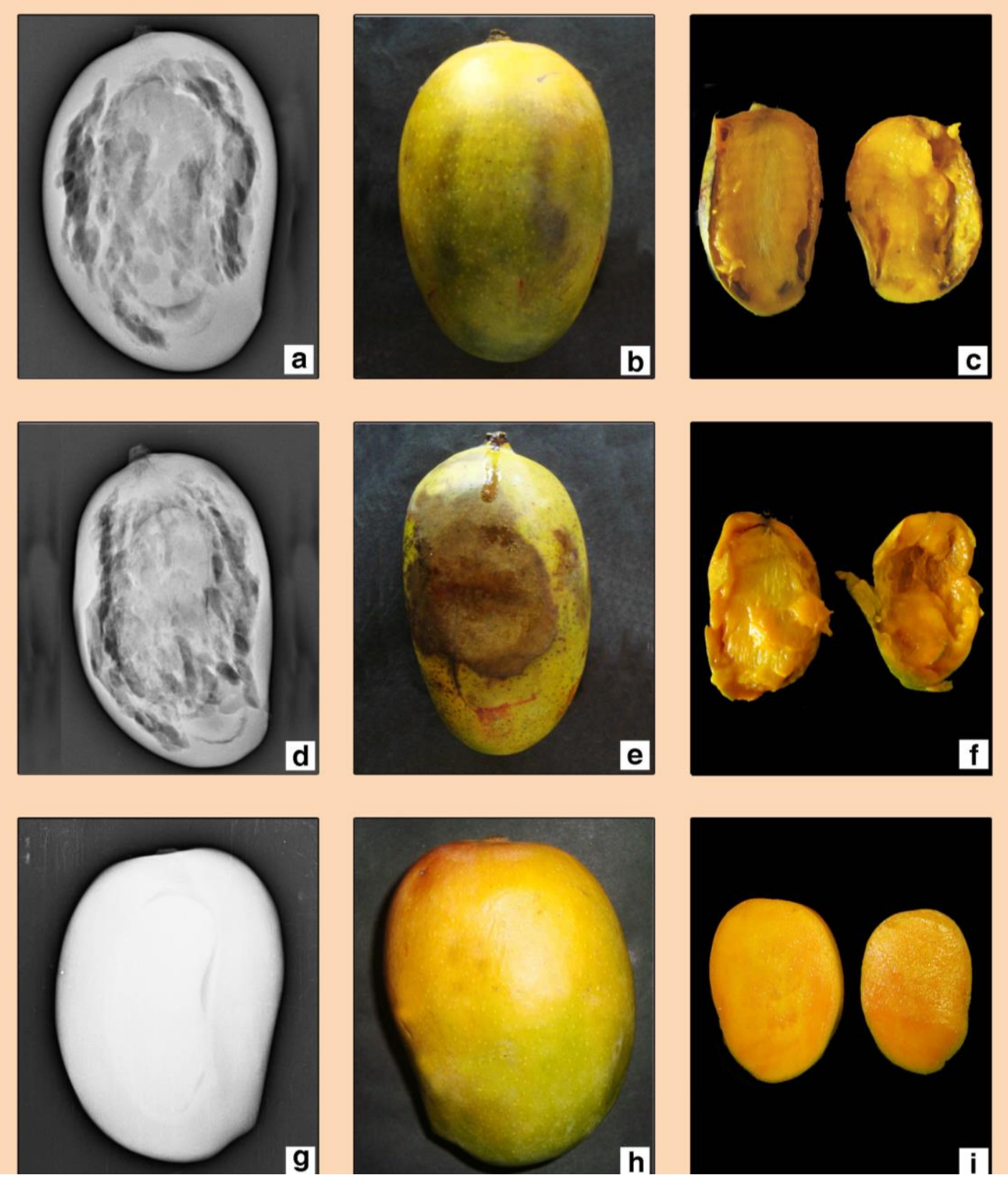

Fig. 2 - X-ray scanning of anthracnose (Colletotrichum rot) of mango fruit. a. X-ray image 4 days post-inoculation, b. uncut fruit 4 days post-inoculation, c. cut fruit 4 days post-inoculation, d. X-ray image 5 days post-inoculation, e. uncut fruit 5 days post-inoculation, f. cut fruit 5 days postinoculation, g. X-ray image of healthy fruit, h. Healthy uncut mango, i. Healthy cut fruit. 

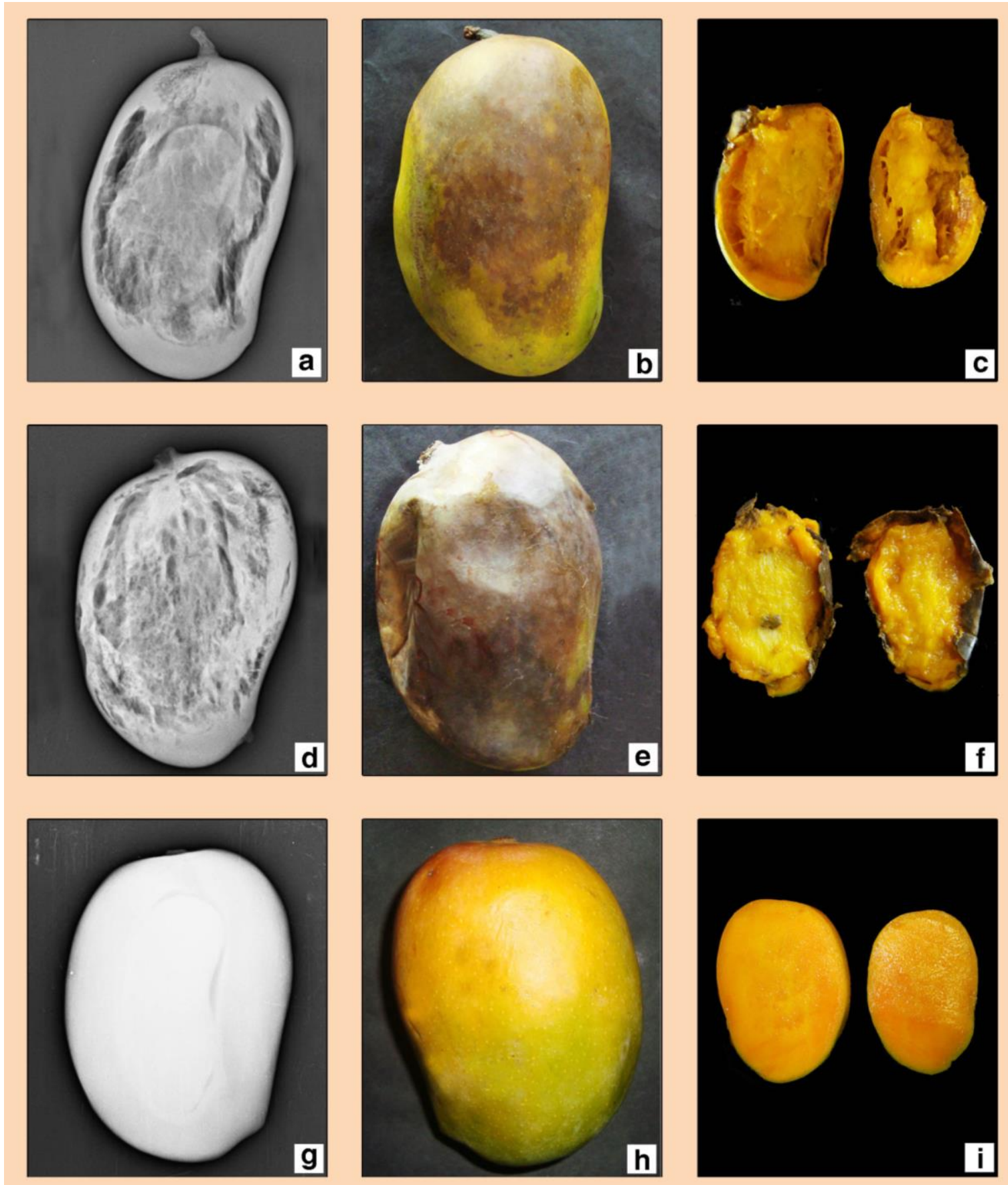

Fig. 3 - X-ray scanning of soft rot (Rhizopus rot) of mango fruit. a. X-ray image 4 days postinoculation, b. uncut fruit 4 days post-inoculation, c. cut fruit 4 days post-inoculation, d. X-ray image 5 days post-inoculation, e. uncut fruit 5 days post-inoculation, f. cut fruit 5 days postinoculation, g. X-ray image of healthy fruit, h. Healthy uncut mango, i. Healthy cut fruit.

\section{Acknowledgements}

The first author is grateful to University Grant Commission (UGC), New Delhi, India for providing financial support. Authors are also thankful to Professor \& Head, Department of Botany, Dr. Babasaheb Ambedkar Marathwada University, Aurangabad (M.S.) India for providing necessary facilities. 


\section{References}

Janave MT. 2007 - Biochemical changes induced due to Staphylococcal infection in spongy Alphonso mango (Mangifera indica L.) fruits. Journal of Crop Science and Biotechnology $10,167-174$.

Thomas P, Saxena S, Chandra R, Rao R, Bhatia CP. 1993 - X-Ray imaging for detecting spongy tissue, an internal disorder in fruits of Alphonso mango (Mangifera indica L.). Journal of Horticulture Science 68, 803-806.

Yang E-C, Yang M-M, Liao L-H, Wu W-Y, Chen T-W, Chen T-M, Lin T-T, Jiang J-A. 2006 Non-destructive quarantine technique - potential application of using X-ray images to detect early infestations caused by Oriental fruit fly (Bactrocera dorsalis) (Diptera: Tephritidae) in fruit. Formosan Entomologist 26, 171-186. 